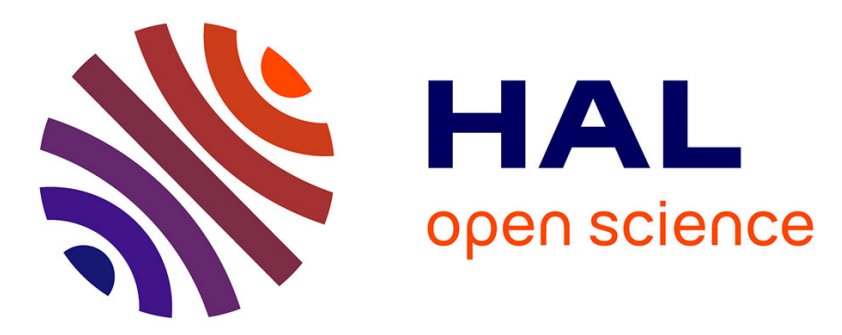

\title{
A dual decomposition strategy of both microbial and phenotypic components for a better understanding of causal claims
}

Gregor P. Greslehner, Maël Lemoine

\section{- To cite this version:}

Gregor P. Greslehner, Maël Lemoine. A dual decomposition strategy of both microbial and phenotypic components for a better understanding of causal claims. Biology and Philosophy, 2020, 35 (1), 10.1007/s10539-019-9708-9 . hal-02285770v2

\section{HAL Id: hal-02285770 \\ https://hal.science/hal-02285770v2}

Submitted on 28 Apr 2020

HAL is a multi-disciplinary open access archive for the deposit and dissemination of scientific research documents, whether they are published or not. The documents may come from teaching and research institutions in France or abroad, or from public or private research centers.
L'archive ouverte pluridisciplinaire HAL, est destinée au dépôt et à la diffusion de documents scientifiques de niveau recherche, publiés ou non, émanant des établissements d'enseignement et de recherche français ou étrangers, des laboratoires publics ou privés. 


\title{
A dual decomposition strategy of both microbial and phenotypic components for a better understanding of causal claims
}

\author{
Gregor P. Greslehner ${ }^{1} \&$ Maël Lemoine ${ }^{1}$ \\ This is a post-peer-review, pre-copyedit version of an article published in Biology \& \\ Philosophy 35:1 (2020). The final authenticated version is available online at: \\ https://doi.org/10.1007/s10539-019-9708-9. \\ Corresponding author: gregor.greslehner@gmail.com \\ ${ }^{1}$ ImmunoConcept, UMR5164, CNRS \& University of Bordeaux, Bordeaux, France \\ ORCID Gregor P. Greslehner: 0000-0002-4072-8229 \\ ORCID Maël Lemoine: 0000-0002-2139-0488
}

\begin{abstract}
In our commentary on Lynch et al.'s target paper (2019, this issue), we focus on decomposition as a research strategy. We argue that not only the presumptive microbial causes but also their supposed phenotypic effects need to be decomposed relative to each other. Such a dual decomposition strategy ought to improve the way in which causal claims in microbiome research can be made and understood.
\end{abstract}

Keywords: Microbiome; Causality; Decomposition; Phenotype

Causal claims of the form 'the microbiome $\mu$ causes phenotype $\varphi$ ' can be frequently found in microbiome research. Lynch et al. (2019, this issue) discuss a number of difficulties with attempts to establish causality between the microbiome and phenotypes of their host. One of the main problems they address - as also recently pointed out by (Bourrat, 2018; Parke et al., 2018; O'Malley and Skillings, 2018) - is the usage of the mass term 'the microbiome' in causal claims, which leaves unclear what the actual causal agents are. Only in exceptional cases will the entirety of microbial entities, components, and activities collectively be responsible for bringing about the phenotype in question. Thus, Lynch et al. emphasize the importance of decomposing "the microbiome into component parts that can be understood independently" $(2019,20)$ for identifying the microbiome's causally relevant components. While we agree with the importance of decomposition, we want to argue for a dual decomposition strategy in 
order to improve causal claims. The following paragraphs address some of the issues related to this dual decomposition strategy.

(i) We suspect that many biologists are already aware of the importance of decomposing the microbiome as a causal entity and just talk about it as a reified unit in non-technical contexts or for heuristic purposes. The authors acknowledge that as a possibility in the final paragraphs of their paper (Lynch et al., 2019, 22). With a charitable reading, undecomposed claims could be understood as placeholder claims: there exist some activities of certain types of microbes within the microbiome that causally contribute in such-and-such a way to the particular aspect of a phenotype. Blaming the chicken sandwich for poisoning before observing the presence of a heavy load of Salmonella (Bourrat, 2018), is, after all, neither naivety nor necessarily a bad strategy. Whether or not researchers are sufficiently aware of the problems accompanying such preliminary claims, the real issue is how to get from these provisional, coarse-grained claims about "the microbiome" to more accurate claims about the causally relevant factors within the microbiome.

(ii) Another, neglected, problem for microbiome researchers who do decompose the microbial components is to also decompose the phenotype into relevant entities and activities for the causal claims they are trying to make. Not only the chicken sandwich, but also the signs and types of food poisoning should be distinguished for the relevant claim that "Salmonella causes food poisoning". This remark emphasizes a different problem for the field of microbiome research: researchers trying to produce new knowledge in a field are specialized in this field. Usually, they rely on established, accepted knowledge in other fields, both out of cautiousness and non-competence. Those who study the phenotype normally do not hypothesize any bold decomposition of the microbiome, and vice versa.

(iii) While researchers might already be aware of the need to decompose the microbiome in order to understand their causal implications, the methodological and disciplinary boundaries might be forcing them into certain frameworks. For instance, the majority of microbiome studies uses sequence data to provide a taxonomic profile based on 16S rRNA. Microbiomewide association studies have focused on linking taxonomic composition to diseases (Gilbert et al., 2016). However, with researchers increasingly realizing that looking at taxonomic composition based on $16 \mathrm{~S}$ rRNA sequencing might not the best way to identify the explanatorily relevant causal factors within the microbiome, they can benefit from a conceptual clarification of how to decompose microbiome structure and function. Taxonomic and genomic profiling remain the major approaches in the field, but the call for alternative techniques and approaches is getting louder, e.g., (Ellegaard and Engel, 2016). Pre-existing ways of decomposing microbiome and phenotypes independently from each other might not be enough. A conceptual clarification of compositional, functional, outcome-oriented, and causal core aspects (Lynch et al., 2019, 11) of microbiomes will certainly enrich the debate in this field. While some ways of decomposition exist for certain phenotypes (e.g., "hallmarks" of cancer (Hanahan and Weinberg, 2000), aging (López-Otín et al., 2013), etc.), there is no such standard for obesity, mental health, or even something seemingly simple as ulcers. In some cases, a phenotype is clear-cut enough not to require any further conceptual decomposition, but in many other cases, decomposition of the phenotype contributes a lot to the clarification needed to understand the causal role of the (decomposed) microbiome.

(iv) Not only do phenotypic effects often have to be also decomposed besides their pre- 
sumptive microbial causes, they also need to be decomposed together. The complete, correct formulation is not that 'something in microbiome $\mu$ causes phenotype $\varphi$ ', but rather that 'something in $\mu$ is causally linked to something in a given $\varphi$ '. The point is not only that a causal claim should achieve specificity in the sense of a one-to-one mapping (as Lynch et al. (2019, 7, Box 1) correctly point out), but rather that decomposition must often be equally performed on the putative cause and its putative effect for the relation to be strongly established. Four different situations illustrate that point.

First, some health conditions are semantically the same but may prove biologically different. This view is commonly held in neurobiological psychiatry as regards conditions such as mood disorders (mainly anxiety and depression), typically diagnosed through behavioral and cognitive symptoms whose detection, and maybe also definition, heavily depends on language. What one wants to causally associate with a remarkable feature of the microbiome is not everything that falls under the name 'depression' or 'obesity', but only one of its biologically specific subtypes. The discovery of a possibly important causal factor like dysbiosis should typically lead to the decomposition of a semantically strong, but biologically weak, entity.

Second, there are also various known phenotypes of the same condition. It is not just a matter of continuity of states from leanness to obesity. Indeed, although it is a minor subject of investigation, different phenotypes of obesity may be defined by the morphological distribution of fat in the organism. This difference has also been associated with differences in metabolism, in turn associated to differential cardiovascular risk (Pajunen et al., 2011). Any of these distinctions may prove crucial in the investigation of the causal relationship with the microbiome. This has time and again proven to be the case in other contexts, such as precisely during the discovery of the role of Helicobacter pylori in peptic ulcer, and it is a useful lesson to be drawn from history.

Third, different pathways may lead to the same pathological outcome. All instances of peptic ulcer do not necessarily stem from the same origin, e.g., H. pylori infection, tobacco use, and consumption of non-steroidal anti-inflammatory drugs. Pathways matter to the definition of a health outcome as one disease or distinct forms of a disease: they are not treated with the same therapeutic strategies, and are often not named the same.

Fourth, a disease may consist in the same loss of balance caused by any defect in any of its components or determinants, as is typically the case in peptic ulcer, in obesity, and probably in mood disorders as well. The decomposition of the cause should be associated with the decomposition of the effect - typically a kind of balance - for any causal claim to be precise enough. Moreover, it is often - if not always - only through the discovery of a specific cause, that the balance in question comes to be adequately described.

(v) Finally, we see an opportunity for philosophers to advance the field beyond the twoplace relation of cause and effect. Figure 1 contrasts the provisional picture of un-decomposed causal claims with that of one in which both microbiome and phenotype are decomposed relative to each other. This picture might also be expanded by external common causes and internal causal connections. As soon as the interactions become more complex, resulting in dynamic chains and networks, looking for two-place cause-effect relations might no longer provide the adequate "level of explanation" (Woodward, 2010) or "causal locus" (O'Malley and Skillings, 2018, 251). Dynamic, mechanistic, and non-causal explanations might prove to be useful tools for these very issues. Establishing a causal relation between a microbial and 


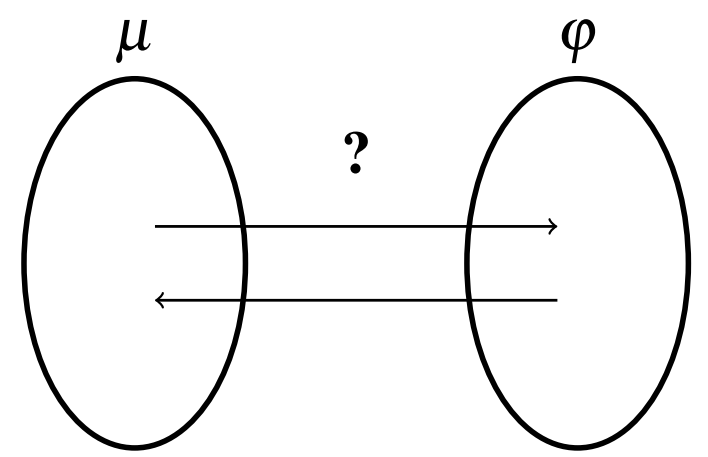

(a)

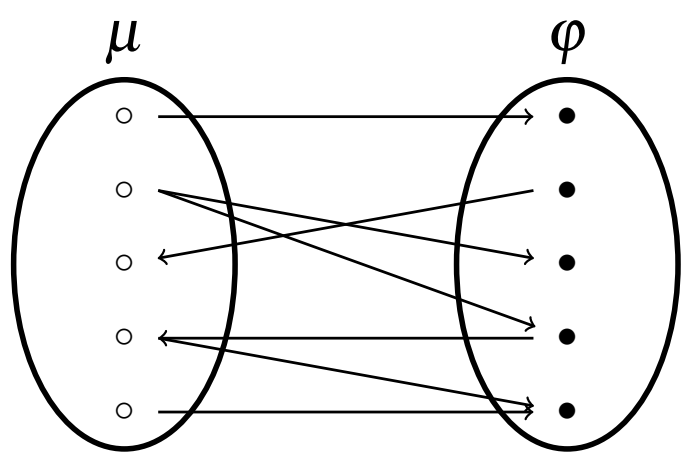

(b)

Figure 1: Causal mapping between microbiome $\mu$ and phenotype $\varphi$, (a) un-decomposed and (b) decomposed.

phenotypic component might be less explanatorily relevant than the mechanisms and topologies of interaction networks, where the identity of individual components becomes less important. That something causes something else will be hard to establish in complex and dynamic interactions. However, it is possible to define clear-cut cause-effect relations between some decomposed microbial and phenotypic components - when they are properly decomposed relative to each other.

A network- and ecology-based perspective needs to be more refined than the kind that Lynch et al. criticize: "this ecological version simply distributes all causality across the whole microbiome" $(2019,20)$. A middle ground position could be to have a specific microbial interaction network that can explain (perhaps non-causally) the aspect of a phenomenon in question, as can be found in philosophical accounts of topological explanations, e.g., (Huneman, 2010; Kostić, 2018). The characteristics of an interventionist account of causal explanation - specificity, stability, and proportionality (Lynch et al., 2019, 7) - will certainly be improved by making causal claims in which microbial and phenotypic causes and effects have been properly decomposed. They might even be better met by other accounts beyond the framework of Koch's postulates and binary causation relations: "although mechanismoriented research can indeed provide causal explanations of particular phenomena, it is clear there are many instances when causal complexity overwhelms straightforward mechanistic accounts" (O'Malley and Skillings, 2018, 254). For a better mechanistic understanding, a dual decomposition of both microbiome and phenotypic effects could at least improve the situation: "Knowledge of a mechanism in the biological sciences is usually more useful for explanation, prediction, and control than merely being able to label something as a cause" (Darden, 2013, 20).

Taken together, we propose a dual decomposition strategy to enrich the debate about a philosophical clarification of concepts and explanatory strategies in microbiome research, which should contribute to a better understanding - and experimental testing - of causal claims about the microbiome. We share this goal with the authors of the target paper (Lynch et al., 2019, this issue) and appreciate the debate that it triggers. 


\section{Acknowledgements}

Many thanks to Thomas Pradeu and the ImmunoConcept group. This project has received funding from the European Research Council (ERC) under the European Union's Horizon 2020 research and innovation programme - grant agreement $n^{\circ} 637647$ - IDEM (P.I.: Thomas Pradeu).

\section{References}

Bourrat, P. (2018). Have causal claims about the gut microbiome been over-hyped? BioEssays, 40(12):1800178. doi:10.1002/bies.201800178.

Darden, L. (2013). Mechanisms versus causes in biology and medicine. In Chao, H.-K., Chen, S.-T., and Millstein, R. L., editors, Mechanism and Causality in Biology and Economics, pages 19-34. Springer. doi:10.1007/978-94-007-2454-9_2.

Ellegaard, K. M. and Engel, P. (2016). Beyond 16S rRNA community profiling: Intra-species diversity in the gut microbiota. Frontiers in Microbiology, 7:1475. doi:10.3389/fmicb.2016.01475.

Gilbert, J. A., Quinn, R. A., Debelius, J., Xu, Z. Z., Morton, J., Garg, N., Jansson, J. K., Dorrestein, P. C., and Knight, R. (2016). Microbiome-wide association studies link dynamic microbial consortia to disease. Nature, 535:94-103. doi:10.1038/nature18850.

Hanahan, D. and Weinberg, R. A. (2000). The hallmarks of cancer. Cell, 100(1):57-70. doi:10.1016/S0092-8674(00)81683-9.

Huneman, P. (2010). Topological explanations and robustness in biological sciences. Synthese, 177(2):213-245. doi:10.1007/s11229-010-9842-z.

Kostić, D. (2018). Mechanistic and topological explanations: an introduction. Synthese, 195(1):1-10. doi:10.1007/s11229-016-1257-z.

López-Otín, C., Blasco, M. A., Partridge, L., Serrano, M., and Kroemer, G. (2013). The hallmarks of aging. Cell, 153(6):1194-1217. doi:10.1016/j.cell.2013.05.039.

Lynch, K. E., Parke, E. C., and O’Malley, M. A. (2019). How causal are microbiomes? A comparison with the Helicobacter pylori explanation of ulcers. Biology \& Philosophy, forthcoming, page numbers refer to the version available at http://tinyurl.com/ BIPH-Lynch [accessed April 22, 2019].

O’Malley, M. A. and Skillings, D. J. (2018). Methodological strategies in microbiome research and their explanatory implications. Perspectives on Science, 26(2):239-265. doi:10.1162/POSC_a_00274. 
Pajunen, P., Jousilahti, P., Borodulin, K., Harald, K., Tuomilehto, J., and Salomaa, V. (2011). Body fat measured by a near-infrared interactance device as a predictor of cardiovascular events: The FINRISK'92 cohort. Obesity, 19(4):848-852. doi:10.1038/oby.2010.236.

Parke, E. C., Calcott, B., and O'Malley, M. A. (2018). A cautionary note for claims about the microbiome's impact on the "self". PLoS Biology, 16(9):e2006654. doi:10.1371/journal.pbio.2006654.

Woodward, J. (2010). Causation in biology: stability, specificity, and the choice of levels of explanation. Biology \& Philosophy, 25(3):287-318. doi:10.1007/s10539-010-9200-z. 